\title{
Radial Numerical Integrations Based On The Sinc Function
}

\author{
Valéry Weber* and Claude Daul \\ Department of Chemistry, University of Fribourg, Fribourg, Switzerland. \\ Richard Baltensperger \\ Department of Mathematics, University of Fribourg, Fribourg, Switzerland.
}

(Dated: August 13, 2004)

\begin{abstract}
An algorithm for the generation of adaptive radial grids used in density functional theory or quantum chemical calculations is described. Our approach is general and can be applied for the integration over Slater or Gaussian type functions with only minor modifications. The relative error of the integration is fully controlled by the algorithm within a specified range of exponential parameters and for a given principal quantum number.

PACS numbers: 71.15.-m; 02.60.Jh; 31.15.Ew
\end{abstract}

Keywords: Numerical quadrature; sinc Function; Molecular Integral

*Electronic address: valery . weber@unifr.ch 


\section{INTRODUCTION}

$A b$-initio quantum chemistry of polyatomic systems has usually relied on fast evaluation of analytic formulas, made possible by the representation of radial atomic orbitals as linear combinations of several Gaussian basis functions. This technique was first introduced by Boys [1] and developed into highly efficient algorithms such as the McMurchie-Davidson method [2], Obara-Saika method [3, 4] and the Rys quadrature [5]. However the increasingly important density functional theory (DFT) requires systematic evaluation of complicated integrals for which there are no basis sets that allow simple closed formulas, but whose integrals are easily evaluated at arbitrary points in space. The first attempts to evaluate those integrals were based on a partitioning of space into muffin-tin shaped regions or to use Monte-Carlo methods based on a smart sampling of the integration points (Diophantine method). In 1988 Becke [6] had the idea to use the resolution of identity $\sum_{i} w_{i}(\mathbf{r})=$ 1 , which is well-known in the mathematical community and proposed the partitioning of polyatomic integrals into single-center components, each of which being evaluated using spherical coordinates. More elaborate schemes have been introduced since then: e.g. te Velde and Baerends [7], Murray, Handy and Laming [8], Treutler and Ahlrichs [9] and recently Gill and Chien [10]. Nevertheless, it seems that the errors introduced by the radial integration are still unacceptably large in many applications and are not easily controlled by the user.

However, a better choice would be an adaptive integration scheme which automatically generates the grid for a given accuracy. Thus the grids is automatically adapted to the basis set chosen for the calculation and therefore reflects the shell structure of the atoms. A heavy atom with more electrons requires a larger grid. Also a larger basis set causes the generation of a larger grid for a given tolerance. An early attempt for the construction of an adaptive grid generator has been proposed by Andzelm and Wimmer [11]. Recently PérezJordá, Becke and San-Fabián [12] presented an automatic numerical integration technique for molecules. They concluded that the available fixed grids are still neither flexible nor effective enough for current calculations. Termath et al [13] have used an adaptive integration scheme to generate optimized grid for their DFT molecular dynamics simulations. Krack and Koster [14] have proposed a fully adaptive molecular grids generator. In their work they have determined the order of the radial quadrature by an empirical interpolation formula 
and the overlap matrix has been chosen as generating function for the angular grid. We can also mention the hierarchical cubature $(\mathrm{HiCu})$ by Challacombe [15]. This interesting method achieves linear scaling computation of the exchange-correlation matrix so important in DFT. Hierarchical cubature combines $k$-dimensional search tree with adaptive numerical integration involving an entirely Cartesian grid.

Lindh et al. [16] published in 2001 an article proposing a quadrature scheme for Gaussian Type Orbitals (GTO) with exponents $\alpha \in\left[\alpha_{m i n}, \alpha_{m a x}\right]$. Our purpose in this work is to present an extension of this idea for Slater Type Orbitals (STO). We describe and justify the particular choice of integration variables and integration formulas, which together constitute the solution to the problem at hand: i.e. DFT. There are indeed a growing number of computational chemists today who are using the ideas of DFT to calculate molecular properties. At the simplest level the Hohenberg-Kohn [17] theorem and the Kohn-Sham [18] theories state that given an appropriate density $\rho(\mathbf{r})$, the exchange, correlation and kinetic energy correction contributions to the energy are given by

$$
I=\int_{\mathbb{R}^{3}} F(\rho(\mathbf{r}), \nabla \rho(\mathbf{r}), \ldots) d^{3} r
$$

where $F$ is a function of the density $\rho(\mathbf{r})$. Most certainly $F$ is of such a complicated form that it is not possible to calculate the integral analytically. It is known for example that a good approximation to the exchange contribution is proportional to $\rho^{4 / 3}(\mathbf{r})$, and more accurate forms are more complicated. As far as is known $F$ is differentiable almost everywhere. The behavior of $F$ is governed by the behavior of $\rho(\mathbf{r})$, which for nearly all molecules has cusp shaped maxima at the nuclei and decreases to zero as $|\mathbf{r}| \rightarrow \infty$. The integral $I$ has normally been calculated by numerical quadrature and the importance of doing this efficiently cannot be over-estimated since it is usually the most time consuming step in molecular applications. Many workers have considered and used molecular grid quadrature over the years. In the early days Boys and Rajagopal [19] gave a scheme, which apportioned molecular space into atomic parts. Boys and Handy [20] used this idea in their trans-correlated studies. Such grid points arose from solving a set of diophantine equations, a method originally due to Haselgrove [21]. More recently Friesner's [22] success in his pseudo-spectral method depended upon the introduction of the molecular grids, which were again atom based. He used radial Chebyshev points and small angular grids. Baerends and co-workers have used the method proposed by Ellis and Painter [23]. Boerrigter et al [24] later found that Gauss- 
Legendre quadrature [25] in the $r, \theta$ and $\phi$ directions gave superior convergence as the number of quadrature points increased. Becke [6] gave a straightforward partition of molecular space into overlapping atomic fragments (fuzzy cells). He recommended the use of GaussChebyshev quadrature [26] in radial direction and Stroud-Lebedev quadrature [27-30] for the angular integration. Andzelm and Wimmer [31] use this approach in their Dgauss program. Numerical integration has a number of advantages in electronic structure calculations, apart from that it provides a means of evaluating otherwise intractable integrals: (i) It is in principle easy to apply to all integrals, such as matrix elements of the operators in the one-electron Schrödinger equation. Moreover numerical integration lends itself naturally to efficient vectorization and parallelization. (ii) As the requirement of analytic integrability is removed, one is free in the choice of basis functions (Slater-type orbitals, numerical atomic functions, etc.). The difficulty to attain high accuracy has been a drawback of 3D integration methods. It has been demonstrated that using the methods described next, it is possible to achieve arbitrarily high accuracy. It is fair to say, however, that very high accuracy requires many integration points. An integration formula, in the volume $V$, is defined by $N$ points $\mathbf{r}_{i}$ and weights $w_{i}$ such that

$$
\int_{V} f(\mathbf{r}) d^{3} r \approx \sum_{i=1}^{N} f\left(\mathbf{r}_{i}\right) w_{i} .
$$

In Newton-Cotes-type formulas (Trapezoidal rule, Simpson's rule, etc.) the step size between two successive grid points is constant. The advantage of this being an easy implementation of a quality controlled step size adaptation like e.g. in Romberg's [25] method. Another advantage of constant step size methods is the exploitation of Euler-MacLaurin's rule according to which it is possible to expand the integration error in a series of odd derivatives at the end points. The idea is to find a variable transformation such that these odd derivatives vanish constantly at the end points. In Gaussian-type formulas the function $f$ is implicitly approximated by a finite expansion in polynomials over the region of integration. The degree of precision is $d$ if the approximation of $f$ is exact for all polynomials of degree smaller or equal to $d$, and not exact for at least one polynomial of degree $d+1$. The practical precision depends then on whether the integrands of interest have rapidly converging expansions in polynomials. With this type of methods an automatic quality controlled step size adaptation is difficult.

In one dimension the problem can be considered solved since the points and weights 
of formulas of any degree for an interval can routinely be computed by well-established standard procedures. In two dimensions formulas of rather high degrees have been published for regular polygons and the surface of the unit sphere. However the computation of these formulas is far to be straightforward, and in practice one has to store the points and the weights as fixed data in the program, thus limiting the application to a few available degrees. Moreover, for regions that are not affinely related to those mentioned, a different solution has to be found. In three and more dimension the situation is even worse: only for a small number of special regions, like the $n$-simplex, the $n$-cube, and the $n$-sphere, a few formulas are known.

The integral (1) is widely encountered in quantum chemistry and can be rewritten in a general form as

$$
I=\int_{\mathbb{R}^{3}} f(\mathbf{r}) d^{3} r
$$

and it is generally convenient to transform (3) into spherical polar coordinates to obtain the radial integral

$$
I=\int_{0}^{\infty} g(r) r^{2} d r
$$

where the factor $r^{2}$ is the Jacobian arising from the coordinate transformation and

$$
g(r)=\int_{0}^{\pi} \int_{0}^{2 \pi} f(\mathbf{r}) \sin \theta d \phi d \theta
$$

is the spherical average of $f(\mathbf{r})$.

As a model for the integration scheme, we will, along this work, study the integration of the functions

$$
\begin{gathered}
\int_{0}^{\infty} u^{m+2} e^{-\alpha u} d u=\frac{\Gamma(m+3)}{\alpha^{m+3}}, \alpha>0 . \\
\int_{0}^{\infty} u^{m+2} e^{-\alpha u^{2}} d u=\frac{\Gamma((m+3) / 2)}{2 \alpha^{(m+3) / 2}}, \alpha>0,
\end{gathered}
$$

where we have introduced the Gamma function $\Gamma(z)=\int_{0}^{\infty} u^{z-1} e^{-u} d u$ for $\Re(z)>0$. In the following we will use the short hand notation

$$
I_{i}=\int_{0}^{\infty} F_{i}(u) d u
$$

where $F_{i}(u)=u^{m+2} e^{-\alpha u^{i}}$ and $i=1,2$.

In the following sections we present a method for the evaluation of radial integrals over either Slater type orbital (STO) or Gaussian type orbital (GTO) based on the sinc function. 
In the Section II a brief introduction to the sinc function is given, then in Section III the method is presented followed by a study for each functions STO and GTO. In Section IV, practical aspects are discussed and some examples are given to demonstrate the efficiency of the method. Finally we make some conclusive remarks.

\section{THE $S I N C$ FUNCTION}

Let $f$ be a complex valued function on the real axis, and suppose we would like to interpolate $f$ between a bi-infinite sequence of equidistant points on $\mathbb{R}$. Without loss of generality, we can assume these interpolation points to be

$$
x_{k}=k h, \quad-\infty<k<\infty, \quad h>0 .
$$

Assuming that the series converge, the interpolation problem is most simply solved by the (Whittaker) cardinal function

$$
C(f, h)(x):=\sum_{k=-\infty}^{\infty} f_{k} S(k, h)(x),
$$

where $S(k, h)$ is given by $S(k, h)(x):=S\left[\frac{\pi}{h}\left(x-x_{k}\right)\right]$ and $S(x)$ is the so called sinc-function

$$
S(x):=\frac{\sin (x)}{x}
$$

and $f_{k}=f\left(x_{k}\right)$. Since $S(0)=1$ and $S(k \pi)=0$ for all nonzero integers $k, C(f, h)$ interpolates $f$ between the $x_{k}$ (as $S(k, h)(x)$ is a translation (up to a factor) of $S(x)$ ).

Interesting features of the cardinal function are its ease of being differentiated and integrated and therefore its ability to approximate the derivative of integrals of an unknown function $f$. In the differentiation case, the derivative of the unknown function at a point $x$ is approximated as

$$
f^{\prime}(x) \approx C(f, h)^{\prime}(x)=\sum_{k=-\infty}^{\infty} f_{k} S(k, h)^{\prime}(x) .
$$

More interesting in the present settings: if the series $C(f, h)$ converges uniformly, one has the following approximation for the integration of $f$ :

$$
\begin{aligned}
\int_{-\infty}^{\infty} f(x) d x & \approx \int_{-\infty}^{\infty} \sum_{k=-\infty}^{\infty} f_{k} S(k, h)(x) d x \\
& =\sum_{k=-\infty}^{\infty} f_{k} \int_{-\infty}^{\infty} S(k, h)(x) d x
\end{aligned}
$$


A straightforward computation leads to $\int_{-\infty}^{\infty} S(k, h)(x) d x=h$ and hence

$$
\int_{-\infty}^{\infty} f(x) d x \approx h \sum_{k=-\infty}^{\infty} f_{k}
$$

which is nothing but the trapezoidal integration rule.

In the present article, we want to approximate the integral of functions which are defined on the semi-infinite interval $[0, \infty)$. By applying the following transformation of variable $u=\psi(x)=e^{x}$, we obtain

$$
\int_{0}^{\infty} f(u) d u=\int_{-\infty}^{\infty} f(u(x)) e^{x} d x
$$

and by using the approximate trapezoidal integration rule, one gets

$$
\begin{aligned}
\int_{0}^{\infty} f(u) d u & =\int_{-\infty}^{\infty} f(u(x)) e^{x} d x \\
& \approx h \sum_{k=-\infty}^{\infty} f\left(e^{x_{k}}\right) e^{x_{k}}
\end{aligned}
$$

We cannot directly use the last (bi-infinite) sum for approximating integrals of function on a computer. One has to do another approximation by truncating the bi-infinite sum

$$
\begin{aligned}
\int_{0}^{\infty} f(u) d u & \approx h \sum_{k=-\infty}^{\infty} f\left(e^{x_{k}}\right) e^{x_{k}} \\
& \approx h \sum_{k=-M}^{N} f\left(e^{x_{k}}\right) e^{x_{k}}
\end{aligned}
$$

Once these two approximations have been made, some practical questions arise:

- How good is this approximation to the integral of the function $f$ ?

- How do we select the step size $h$, the limiting terms $M$ and $N$ in the sum?

- Is it possible to design an automatic procedure to obtain a certain relative precision in the numerical computation of a given integral?

In this work, we want to use this classical trapezoidal integration rule to approximate the integral of STO as well as of GTO. 


\section{NUMERICAL INTEGRATION}

In order to answer the questions raised in the preceding section, we shall review some important results given by Lund and Bowers [32] or by Stenger [33].

Let us first define the domain

$$
D_{W} \equiv\left\{w \in \mathbb{C}: w=r e^{i \theta},|\theta|<d \leq \pi / 2, r \in \mathbb{R}^{+}\right\} .
$$

which is the domain presented on the left hand side of Figure 1. Our aim is to compute (numerically and as precisely as possible) the integral $\int_{0}^{\infty} f(u) d u$ of a given function $f$ which is analytic in the domain $D_{W}$.

The following theorem stated in Lund and Bowers [32] and Stenger [33] yields an integration rule (16) (namely a truncated trapezoidal rule), a stepsize $h$ (22), a limiting term $N(21)$ and an (absolute) error estimate (23)

Theorem 1 Let $f$ be analytic in $D_{W}$ where the angle of the wedge opening is $d(0<d \leq$ $\pi / 2)$,

$$
\begin{aligned}
\int_{-d}^{d}\left|f\left(r e^{i \theta}\right)\right| r d \theta=\mathcal{O}\left(|\ln r|^{a}\right), & r \rightarrow 0^{+}, \infty \\
& 0 \leq a<1,
\end{aligned}
$$

and

$$
\lim _{r \rightarrow 0^{+}, R \rightarrow \infty} \int_{r}^{R}\left|f\left(\rho e^{i d}\right)\right| d \rho<\infty .
$$

Further assume that there are positive constants $\gamma, \eta$, and $C$ so that

$$
|f(u)| \leq C \begin{cases}u^{\gamma-1}, & u \in(0,1), \\ u^{-\eta-1}, & u \in[1, \infty) .\end{cases}
$$

If the selections

$$
N=\left[\left|\frac{\gamma}{\eta} M+1\right|\right]
$$

and

$$
h=\left(\frac{2 \pi d}{\gamma M}\right)^{1 / 2} \leq \frac{2 \pi d}{\ln 2}
$$

are made, then the absolute error ( $A E)$ is given by:

$$
\begin{aligned}
A E & =\left|\int_{0}^{\infty} f(u) d u-h \sum_{k=-M}^{N} e^{k h} f\left(e^{k h}\right)\right| \\
& =\mathcal{O}(\exp (-\sqrt{2 \pi d \gamma M})) .
\end{aligned}
$$


Where [.] stands for the nearest integer value. The selection of $N$ in (21) is for technical accuracy as mentioned by Lund and Bowers[32].

In general, the exact calculation of integrals (18) and (19) is quite difficult and one does not do their calculations before implementing the rule (23). Rather, if the integrand $f$ is analytic in a sector of the right half plane (angle of opening $d$ ) and integrable on rays in this sector, then this angle $d$ defines, via (22), a suitable mesh size. The exponents in (20) refer only to the behavior of $f$ on the half line.

Once $\gamma, \eta, d$ and $M$ are known all other quantities in the theorem can be computed and one obtain a practical and fairly simple quadrature rule! $\gamma, \eta$ and $d$ depend on the function one wants to integrate and $M$ depends on the desired precision one wishes to achieve.

The performance of the preceding $(z=\phi(w)=\ln (w))$ quadrature rule may be improved with respect to the number of function evaluations. Assume the integrand exhibits exponential decay at infinity

$$
|f(u)| \leq C\left\{\begin{array}{l}
u^{\gamma-1}, u \in(0,1), \\
e^{-\eta u}, u \in[1, \infty) .
\end{array}\right.
$$

Then, the upper limit of the number of integration nodes can be significantly reduced by selecting

$$
N=\left[\left|\frac{1}{h} \ln \left(\frac{\gamma}{\eta} M h\right)+1\right|\right] .
$$

The exponential convergence of the approximated sum is maintained with this alternative selection of $N$ as verified by Lund and Bowers [32].

In (25), $N$ may become negative for very large values of $\eta$. An elementary computation yields $N=0$, when $\eta=\gamma M h e^{h}$. By slightly modifying the transformation $z=\phi(w)=\ln (w)$ to $z=\phi(w)=\ln (w / c)$ with a given constant $c>0$, equation (21) is changed to

$$
N=\left[\left|\frac{1}{h} \ln \left(\frac{\gamma}{\eta c} M h\right)+1\right|\right]
$$

and hence the parameter $c$ can be chosen according to

$$
c=\frac{\gamma M h e^{h}}{\eta}
$$

and the numerical integration rule will be given by

$$
\text { ch } \sum_{k=-M}^{N} e^{k h} f\left(c e^{k h}\right) .
$$

This slight modification of the integration rule will help us to control the (relative) error growth in the computation of STO and GTO integrals. 


\section{A. Slater type orbital}

Remember that we want to compute numerically

$$
I_{1}=\int_{0}^{\infty} F_{1}(u) d u
$$

with $F_{1}(u)=u^{m+2} e^{-\alpha u}$. In this case, one has clearly

$$
\left|F_{1}(u)\right| \leq C_{1}\left\{\begin{array}{l}
u^{\gamma-1}, u \in(0,1), \\
e^{-\eta u}, u \in[1, \infty),
\end{array}\right.
$$

and the angle of the wedge opening $d_{1}$ is $\pi / 2$.

Therefore, the step size $h_{1}$, the parameter $c_{1}$ as well as the upper limit $N_{1}$ are given by

$$
\begin{aligned}
h_{1} & =\pi \sqrt{\frac{1}{M_{1}(m+3)}}, \\
c_{1} & =\frac{M_{1}(m+3) h_{1} e^{h_{1}}}{\alpha_{\max }} \text { and } \\
N_{1} & =\left[\left|\frac{1}{h_{1}} \ln \left(\frac{M_{1}(m+3) h_{1}}{\alpha_{\min } c_{1}}\right)+1\right|\right] .
\end{aligned}
$$

Remember that we want to compute the integral of $F_{1}$ for a fixed $m$ and a range of $\alpha \in$ $\left[\alpha_{\min }, \alpha_{\max }\right]$, hence one has to take $\alpha_{\max }$ for $c_{1}$ and $\alpha_{\min }$ for $N_{1}$.

\section{B. Gaussian type orbital}

Here we want to compute numerically

$$
I_{2}=\int_{0}^{\infty} F_{2}(u) d u
$$

with $F_{2}(u)=u^{m+2} e^{-\alpha u^{2}}$. In this case, one has

$$
\left|F_{2}(u)\right| \leq C_{2} \begin{cases}u^{\gamma-1}, & u \in(0,1), \\ e^{-\eta u^{2}}, & u \in[1, \infty)\end{cases}
$$


and the angle of the wedge opening $d_{2}$ is $\pi / 4$. Presently the exponential decay at infinity is greater than in the STO case and the different parameters are given by:

$$
\begin{aligned}
h_{2} & =\frac{h_{1}}{\sqrt{2}} \\
c_{2} & =e^{h_{2}} \sqrt{\frac{M_{2}(m+3) h_{2}}{\alpha_{\max }}} \text { and } \\
N_{2} & =\left[\left|\frac{1}{2 h_{2}} \ln \left(\frac{M_{2}(m+3) h_{2}}{\alpha_{\min } c_{2}^{2}}\right)+1\right|\right] .
\end{aligned}
$$

The last result can be derived in the same way as in Lund and Bowers [32].

\section{PRACTICAL ASPECTS}

In this section we will illustrate how to obtain a closed relation for a fully adaptive radial grid based on the STO and GTO functions, namely $F_{i}(u)=u^{m+2} e^{-\alpha u^{i}}$, where $i=1$, 2 . All the calculations presented in this article were obtained with double precision floating-point numbers using 64 bits arithmetic.

As the error bound given for the evaluation of the integral (23) cannot be evaluated in an easy way, one has to find an empirical function which will allow us to compute the number of points $M_{i}$ as a function of the desired precision $R E_{i}$ and for a given $m$. For this purpose we have chosen a model function (which is inspired by the error bound (23)) of the type

$$
\begin{aligned}
R E_{i}\left(M_{i}, m\right) & =\frac{A E_{i}\left(M_{i}, m\right)}{\left|I_{i}\right|} \\
& =K_{i}(m) e^{-A_{i}(m) \sqrt{M_{i}}}, \quad i=1,2,
\end{aligned}
$$

$K_{i}(m)$ and $A_{i}(m)$ are the empirical parameters that need to be determined. Here we have supposed that the absolute error $A E_{i}$ is a function of only $M_{i}$ and $m$ and do not depend on the range of exponents $\left[\alpha_{\min }, \alpha_{\max }\right]$. In Figure 2 we have plotted the logarithm of Eq. (33) for the STO function for three different values of $m=0,10$ and 25 as a function of the square root of $M_{i}$. The data have been selected to lie in a relative error range between $10^{-5}$ to about $10^{-14}$. We see that the exponential parametrization is a good model since the exponential decay of the relative error $R E_{i}$ is obeyed. Similar results were obtained for the GTO functions.

Now the dependence in $m$ can be introduced, after a simple inspection of the data sets. A cubic fit of the functions $\ln \left(K_{1}(m)\right), A_{1}(m), \ln \left(K_{2}(m)\right)$ and $A_{2}(m)$ provides good estimates. 
The data and the fits are shown in Figs. 3 and 4 for the STO and GTO functions respectively. It is clear that other kind of fit functions could have been used. In particular a polynomial function in square root of $m$ would have been a suitable choice to fit $A_{1}(m)$ and $A_{2}(m)$ as expected from Eq. (23).

The following cubic fit parameters are obtained for STO

$$
\begin{aligned}
\ln \left(K_{1}(m)\right)= & 0.5114010^{1}+0.8937410^{0} m \\
& -0.2402110^{-1} m^{2}+0.1979810^{-3} m^{3}
\end{aligned}
$$

and

$$
\begin{aligned}
A_{1}(m)= & 0.4925210^{1}+0.4905610^{0} m \\
& -0.1739210^{-1} m^{2}+0.2425510^{-2} m^{3}
\end{aligned}
$$

and for GTO

$$
\begin{aligned}
\ln \left(K_{2}(m)\right)= & 0.3138010^{1}+0.6906810^{0} m \\
& -0.1863510^{-1} m^{2}+0.2721910^{-3} m^{3}
\end{aligned}
$$

and

$$
\begin{aligned}
A_{2}(m)= & 0.3684110^{1}+0.4128110^{0} m \\
& -0.1421110^{-1} m^{2}+0.2297010^{-2} m^{3}
\end{aligned}
$$

for any power $0 \leq m \leq 25$. These empirical parameters can then be used to evaluate the number of points needed to obtain a given accuracy $R E_{i}$ for a given exponent $m$ through the general relation

$$
M_{i}\left(R E_{i}, m\right)=\left(\ln \left(\frac{R E_{i}}{K_{i}(m)}\right) \frac{1}{A_{i}(m)}\right)^{2}, \quad i=1,2 .
$$

In Figs 5, 6 and 7 we have used the fitted functions Eqs. (34-35) to estimate the number of points $M_{1}\left(R E_{1}, m\right)$ needed to integrate the STO function for different accuracies $R E_{1}=$ $10^{-5}, 10^{-10}$ and $10^{-15}$, and for different values of $m=0,10$ and 25 . In some cases, the relative error $R E_{1}$ was "exactly zero" (within the double precision floating-point arithmetic) and for convenience those errors were set to $10^{-18}$. Similar results were obtained for the numerical integration of the GTO function based on the fitted functions (36-37). We can observe a discrepancy of only one order of magnitude in the case where $m=25$ and $R E_{1}=10^{-15}$ (See Fig. 7) when using the fitted functions (34-35). 
TABLE I: Number of grid points $N^{T o t}=N+M+1$ needed to integrate GTO and STO functions within a relative error $R E=10^{-12}$ with the exponent range $\alpha \in\left[10^{-1}, 10^{5}\right]$ for different values of $m$.

\begin{tabular}{ccccc}
\hline \hline & \multicolumn{2}{c}{ GTO } & \multicolumn{2}{c}{ STO } \\
$m$ & Lindh $^{a}$ & Present & Lindh $^{b}$ & Present \\
\hline 0 & 102 & 116 & 91 & 96 \\
1 & 90 & 108 & 87 & 95 \\
2 & 85 & 103 & 86 & 94 \\
10 & - & 92 & - & 98 \\
25 & - & 100 & - & 118 \\
\hline \hline
\end{tabular}

${ }^{a}$ Lindh et al.[16]

${ }^{b}$ Lindh et al.[16] quadrature adapted for STO (See Appendix A).

We also present in Table I the total number of grid points $N^{T o t}(R E, m)=M+N+1$ for a given accuracy $R E=10^{-12}$ and with the exponent range $\alpha \in\left[10^{-1}, 10^{5}\right]$ for different values of $m$. The number of points for the GTO integrations are compared with the results proposed by Lindh et al. [16]. We have also derived the STO equivalent of the Lindh et al. quadrature (See Appendix A).

As we can see in Table I, the total number of points $N^{T o t}(R E, m)$ for a give $R E$ is slightly higher for our method than for Lindh et al. [16] method. However, our method covers a bigger range of $m$ and does not suffer from solving nonlinear equations to find the optimal parameters.

\section{CONCLUSION}

An algorithm for the generation of radial grids that are needed in density functional theory or quantum chemical calculations has been described. From a pure computational point of view it is not trivial to give "useful" expressions for the unknown parameters $K_{1}$ and $K_{2}$ although analytical relations exist [32]! But, we could show that a simple numerical analysis of the behavior of the number of point $M_{i}$ with respect to the relative error and $m$ can lead to a very simple parameterization. We have also shown that these compact 
relations are valid for a large range of relative errors and parameters $m$ up to 25 without affecting the stability of the relative accuracy. Of course, this large range of $m$ covers almost the whole need of computational chemistry. Thus the method introduced in this paper can be used for a large range of exponents $\alpha$ and $m$ with a full control of the relative error in these ranges. It is worth mentioning that the method is not limited to the STO or GTO functions. Thus a family of optimal quadrature formulas for approximating the integral $I=\int_{D} f(x) d x$, in the domain $D$, can be derived [34]. The integrand $f(x)$ may even have singularities at the end points of integration. In a future work adaptive three dimensional grid and Poisson equation solver based on the sinc function will be addressed elsewhere.

\section{ACKNOWLEDGMENTS}

This work is supported by the Swiss National Science Foundation and by the Swiss Office for Education and Science through the European COST Action D14.

\section{APPENDIX A: LINDH BASED QUADRATURE RULE FOR STO FUNCTION}

In this appendix, we follow Lindh [16] and present the important relations needed for the quadrature rule based on the STO function.

$$
R_{D}=\frac{2 \sqrt{2 \pi}}{(m+2) !}\left(\frac{2 \pi}{h}\right)^{m+\frac{5}{2}} e^{-\frac{\pi^{2}}{h}}(1+\mathcal{O}(h)),
$$

This relation (A1) is very similar to the equation (18) given by Lindh [16]. The upper bound of the integral $I_{1}$ can be calculated exactly as

$$
\begin{aligned}
R_{L} & =\frac{\alpha_{m i n}^{m+3}}{(m+2) !} \int_{x_{N}}^{\infty} m^{m+2} e^{-\alpha_{\min } x} d x \\
& =e^{-\alpha_{\min } x_{N}} \sum_{i=0}^{m+2} \frac{x_{N}^{i} \alpha_{\min }^{i}}{i !}
\end{aligned}
$$

and the lower bound is given by

$$
\begin{aligned}
R_{H} & =\frac{\alpha_{\max }^{m+3}}{(m+2) !} \int_{0}^{x_{1}} m^{m+2} e^{-\alpha_{\max } x} d x \\
& =1-e^{-\alpha_{\max } x_{1}} \sum_{i=0}^{m+2} \frac{x_{1}^{i} \alpha_{\max }^{i}}{i !} .
\end{aligned}
$$


Given a specific power $m$ and a required relative error $R E$ the parameter $h$ is found for a given discretization error $R_{D}=R E$. The most diffuse function $\left(\alpha_{\min }\right)$ determines the outermost point $x_{N}$ such that $R_{L}=R E$. The function with the largest exponent $\alpha_{\max }$ determines the point $x_{1}$ such that $R_{H}=R E$. From these parameters we obtain $c=x_{1} /\left(e^{h}-1\right)$ and $N=\ln \left(1+x_{N} / c\right) / h$.

1 S. F. Boys, Proc. Roy. Soc. A200, 542 (1950).

2 L. E. McMurchie and E. R. Davidson, J. Comput. Phys. 26, 218 (1978).

3 S. Obara and A. Saika, J. Chem. Phys. 84, 3963 (1986).

${ }^{4}$ S. Obara and A. Saika, J. Chem. Phys. 89, 1540 (1988).

5 Dupuis, Rys, and King, J. Chem. Phys. 65, 111 (1976).

6 A. Becke, J. Chem. Phys. 88, 2547 (1988).

7 G. te Velde and E. J. Baerends, J. Comput. Phys. 99, 84 (1992).

8 C. W. Murray, N. C. Handy, and G. J. Laming, Mol. Phys. 78, 997 (1993).

9 O. Treutler and R. Ahlrichs, J. Chem. Phys. 102, 346 (1995).

10 P. M. W. Gill and S. Chien, J. Comput. Chem. 24, 732 (2002).

11 J. Andzelm and E. Wimmer, J. Chem. Phys. 96, 1280 (1991).

12 J. Pérez-Jordá and A. Becke, J. Chem. Phys. 100, 6520 (1994).

13 V. Termath and J. Sauer, Chem. Phys. Lett. 255, 187 (1996).

14 M. Krack and A. Köster, J. Chem. Phys. 108, 3226 (1998).

15 M. Challacombe, J. Chem. Phys. 113, 10037 (2000).

16 R. Lindh, P. Malmqvist, and L. Gagliardi, Theor. Chim. Acta 106, 178 (2001).

17 P. Hohenberg and W. Kohn, Phys.Rev B136, 854 (1964).

18 W. Kohn and L. J. Sham, Phys.Rev A145, 1133 (1965).

19 S. F. Boys and P. Rajagopal, Adv. Quantum. Chem. 2, 1 (1965).

20 S. F. Boys and N. C. Handy, Proc. Roy. Soc. A311, 309 (1969).

21 C. B. Haselgrove, Math. Comput. 15, 323 (1961).

22 R. A. Friesner, J. Chem. Phys. p. 3522 (1987).

23 D. E. Ellis and G. S. Painter, Phys. Rev. B22, 887 (1970).

24 P. M. Boerrigter, G. te Velde, and E. J. Baereends, 33, 87 (1988). 
25 W. H. Press, S. A. Teukolsky, W. T. Vetterling, and B. P. Flannery, Numerical Recipies in FORTRAN (Cambridge University Press, Port Chester, NY, 1992).

26 M. Abramowitz and I. E. Stegun, Handbook of Mathematical Function (Dover, New York, 1970).

27 V. I. Lebedev, Zh. Vychisl. Mat. Mat. Fiz. 15, 48 (1975).

28 V. I. Lebedev, Zh. Vychisl. Mat. Mat. Fiz. 16, 293 (1976).

29 V. I. Lebedev, Sibirsk. Mat. Fiz. 18, 132 (1977).

30 V. I. Lebedev and A. L. Skorokhodov, Russ. Acad. Sci. Dokl. Math. 45, 587 (1992).

31 J. Andzelm and E. Wimmer, J. Chem. Phys. 92, 1280 (1992).

32 J. Lund and K. L. Bowers, Sinc methods for quadrature and differential equations (SIAM, 1992).

33 F. Stenger, Numerical methods based on Sinc and analytic functions (Spinger, 1993).

34 K. Sikorski and F. Stenger, ACM Trans. Math. Soft. 10, 140 (1984). 
FIG. 1: The domain $D_{Z}$ and $D_{W}$.
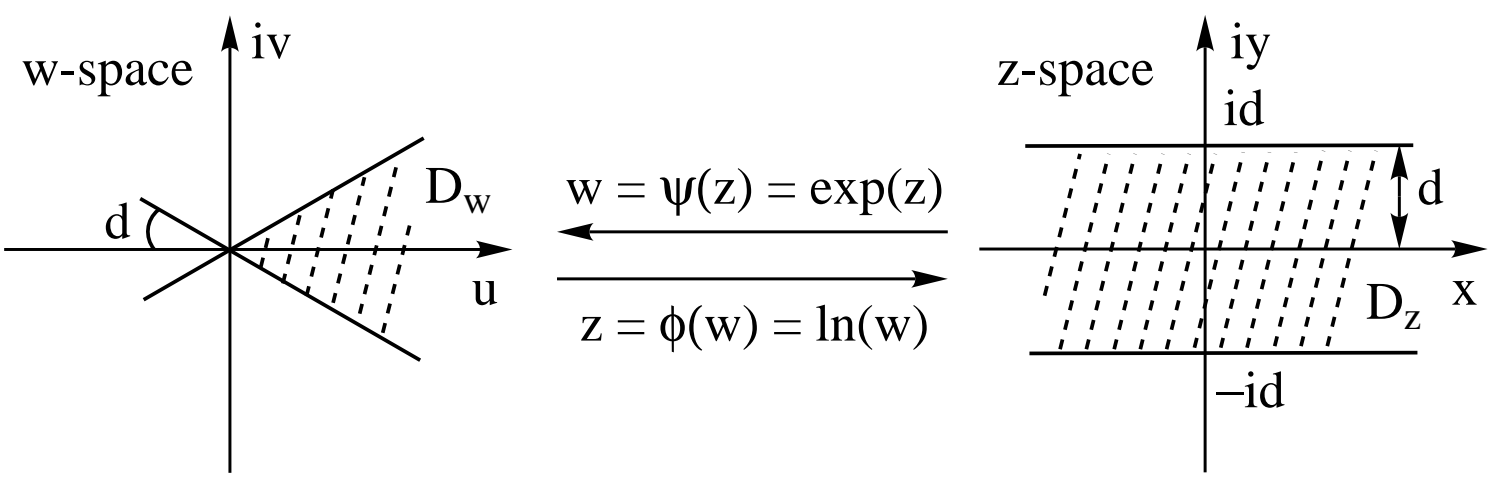

FIG. 2: Linear fits of the data sets $\log \left(E R_{1}\left(M_{1}\right)\right)$ in function of $M_{1}^{1 / 2}$ for different values of $m=0,10$ and 25 .

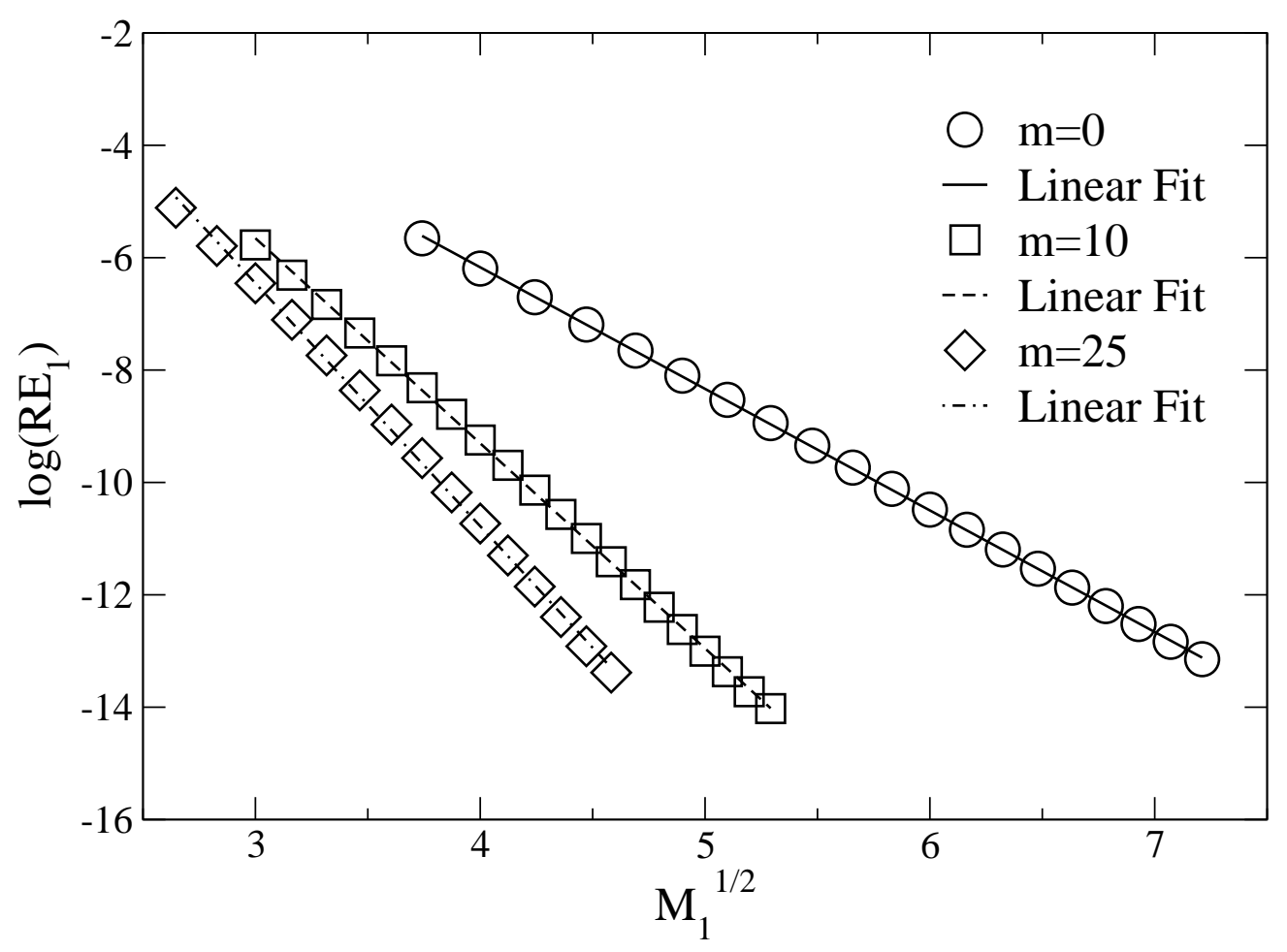


FIG. 3: Cubic fits of the data sets $\ln \left(C_{1}(m)\right)$ and $A_{1}(m)$ in function of $m \in[0,25]$.

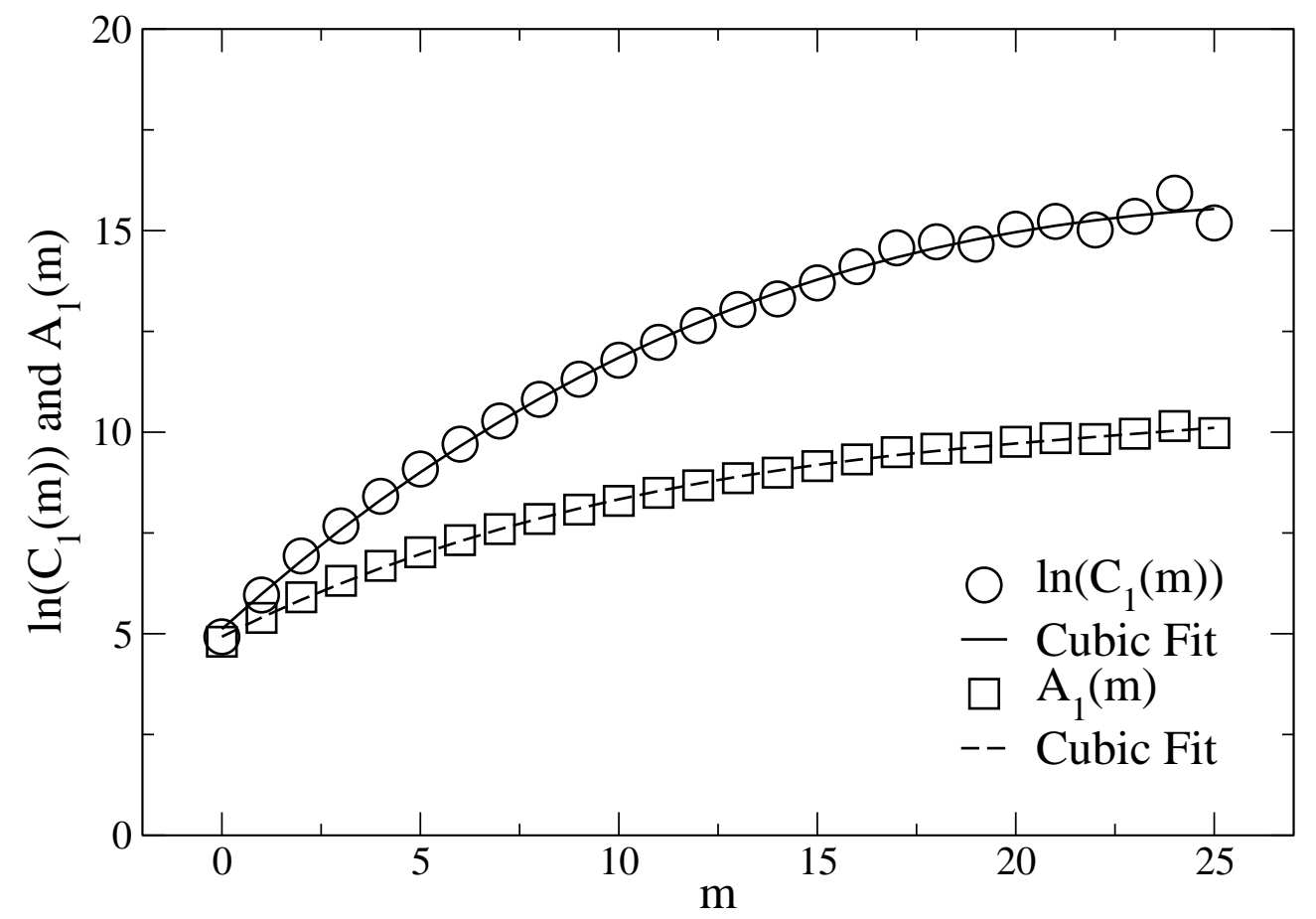


FIG. 4: Cubic fits of the data sets $\ln \left(C_{2}(m)\right)$ and $A_{2}(m)$ in function of $m \in[0,25]$.

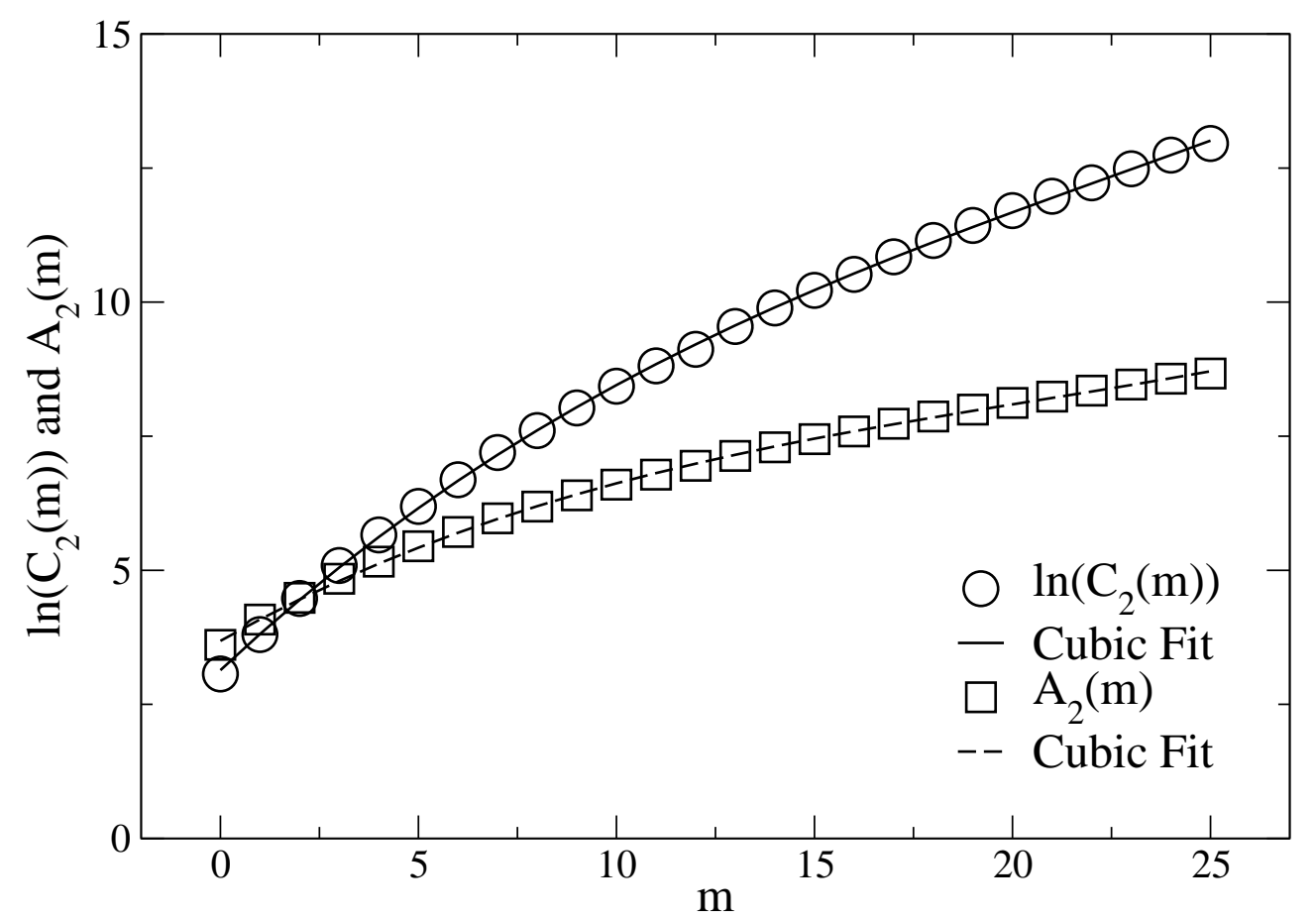


FIG. 5: Logarithm of the relative error $R E_{1}$ as a function of the STO exponent for $m=0$ and $\alpha \in\left[10^{-1}, 10^{-5}\right]$. The number of grid points is given by the fits Eq. (34) and (35) for desired accuracies of $10^{-5}, 10^{-10}$ and $10^{-15}$.

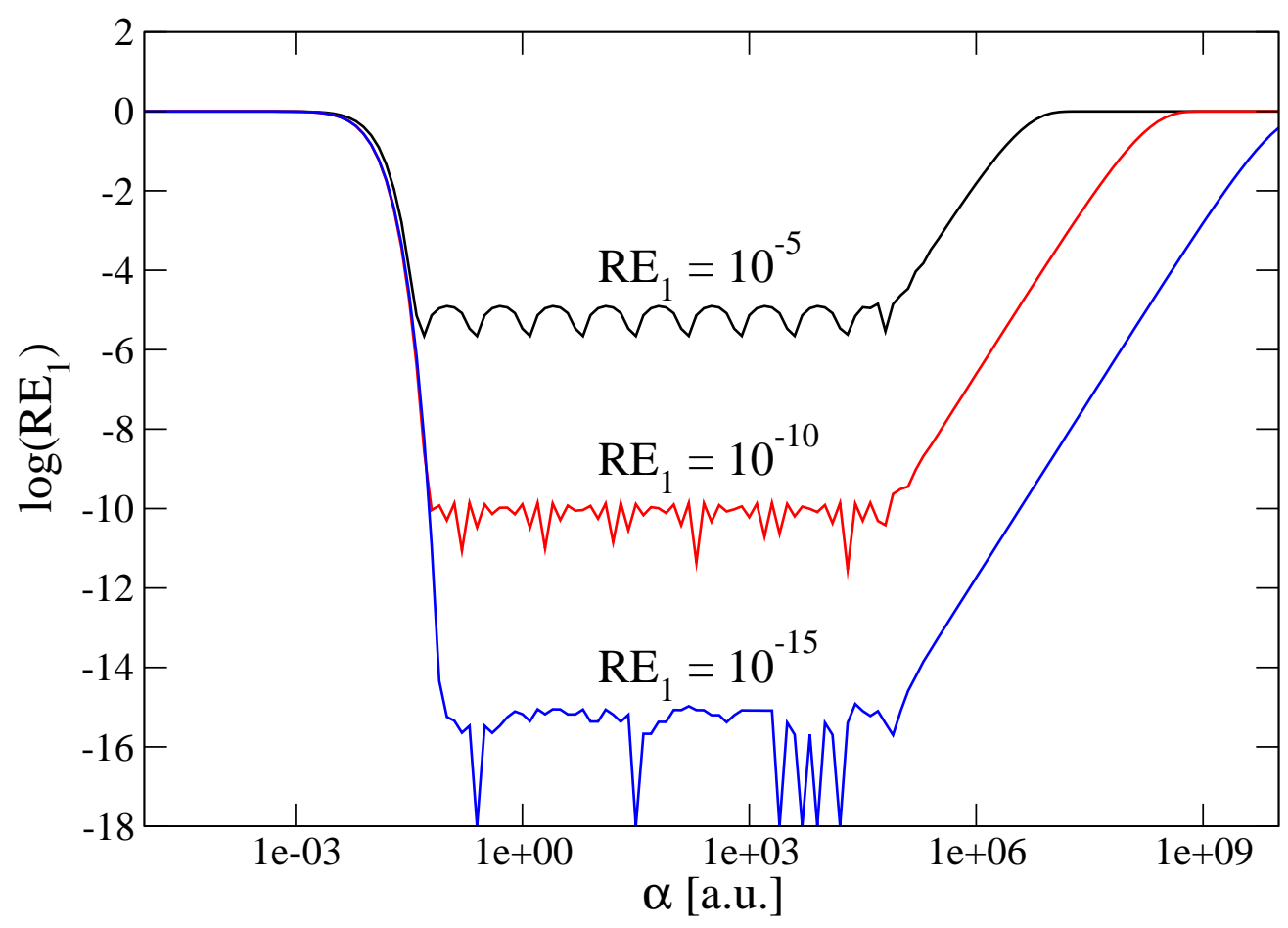


FIG. 6: Logarithm of the relative error $R E_{1}$ as a function of the STO exponent for $m=10$ and $\alpha \in\left[10^{-1}, 10^{-5}\right]$. The number of grid points is given by the fits Eqs. (34) and (35) for desired accuracies of $10^{-5}, 10^{-10}$ and $10^{-15}$.

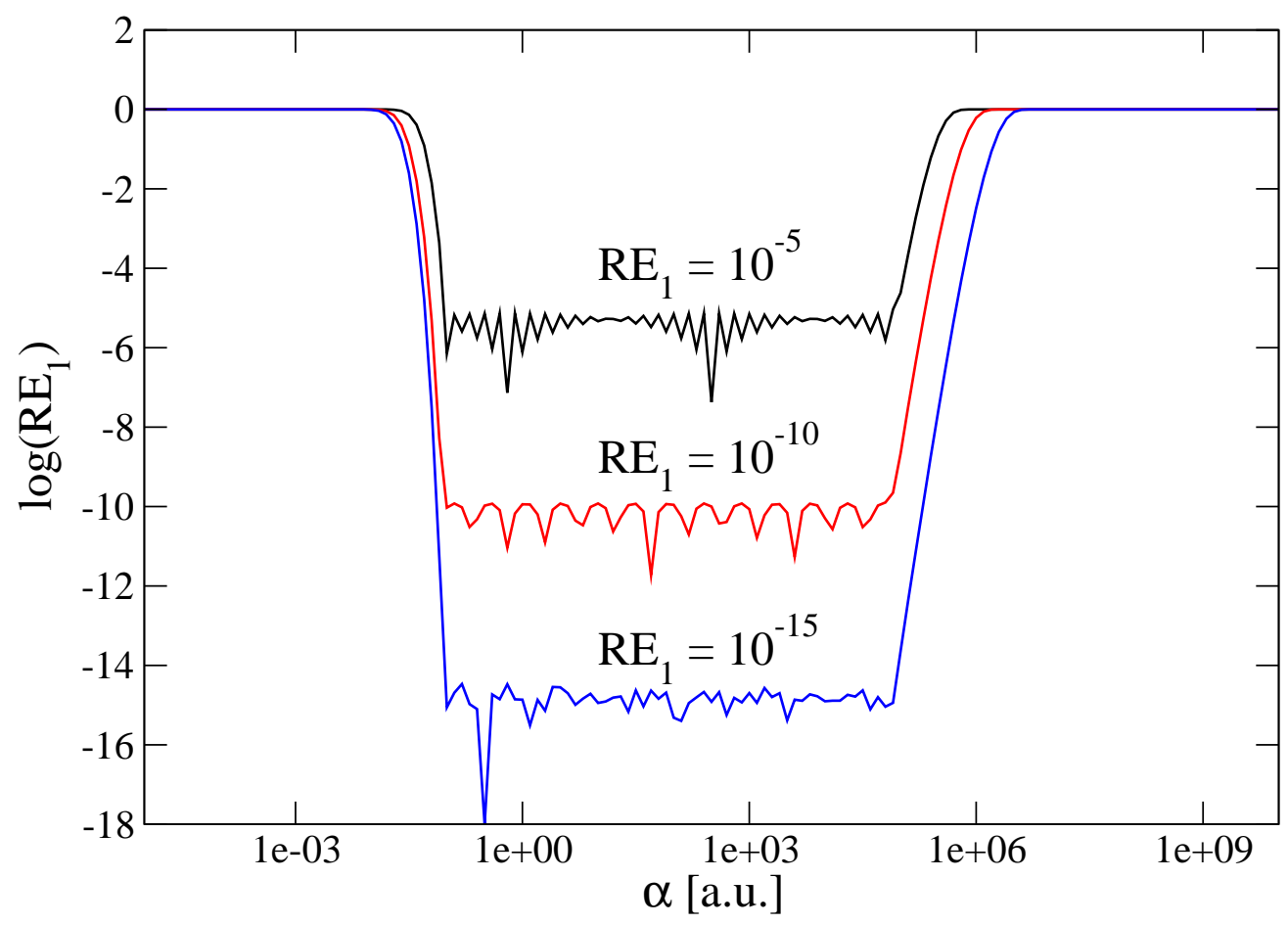


FIG. 7: Logarithm of the relative error $R E_{1}$ as a function of the STO exponent for $m=25$ and $\alpha \in\left[10^{-1}, 10^{-5}\right]$. The number of grid points is given by the fits Eqs. (34) and (35) for desired accuracies of $10^{-5}, 10^{-10}$ and $10^{-15}$.

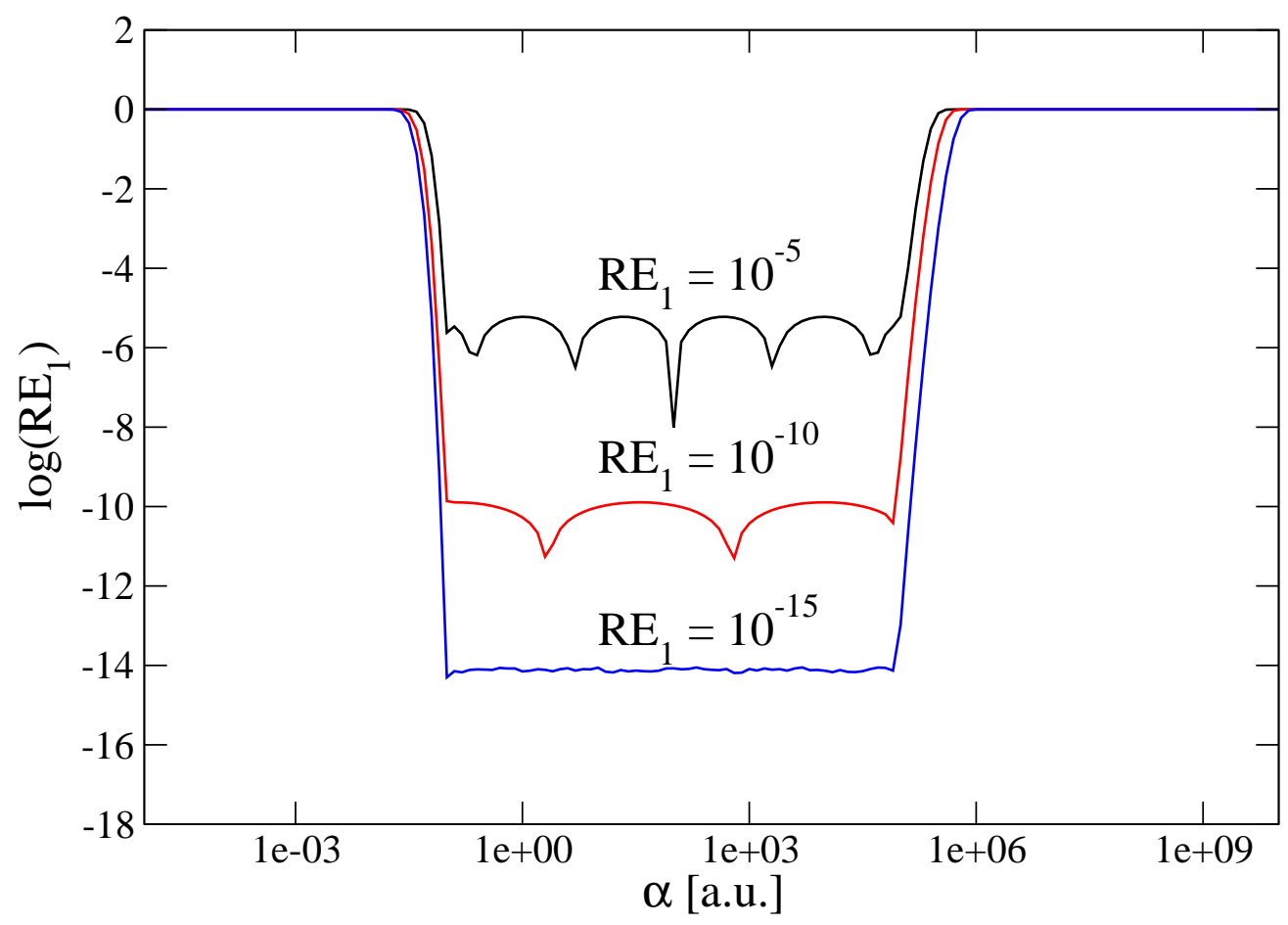

